\title{
Acquisition and Analysis System of the Ballistocardiogram Signal Based on Virtual Instruments
}

\author{
Fangfang Jiang, Xu Wang, Dan Yang, Yu Hao \\ College of Information Science and Engineering, Northeastern University, \\ Shenyang 110819, China \\ geminixy@163.com
}

\begin{abstract}
Keywords: Ballistocardiogram signal, Acquisition and analysis system, Virtual instrument, LabVIEW
\end{abstract}

\begin{abstract}
Ballistocardiogram signal (BCG) is a non-invasive technique for the assessment of the cardiac function. It consists mainly of heart movement and the movement of blood in aorta, arteries, and periphery, which can be used to real-time monitor the heart rate and respiration frequency at home. In our laboratory, a sitting BCG detection chair has been designed successfully, and the acquisition and analysis system based on virtual instruments is proposed in this paper. MATLAB7.0 and LabVIEW8.5 were used to simulate the operational environment, and the results show high efficiency and accuracy in displaying waveform and spectrum, extracting main characteristics of heart rate and respiratory frequency, and alerting when abnormal heart-rate occurs.
\end{abstract}

\section{Introduction}

Heart disease is one of the most serious diseases to threat for human health, and the cardiovascular disease diagnosis and prevention have become the most important issue to the biomedical engineering research. In order to monitor heart state at home conveniently, BCG signal is applied with chair, bed and so on. These hardware detecting equipments have been developed in different countries, but the supporting software environments are not form. Therefore, acquisition and analysis system was designed and realized in this paper by LabVIEW [1].

Virtual instrument technology is the use of high performance modular hardware, combined with efficient and flexible software to complete a variety of test, measurement and automation applications. LabVIEW virtual instrument is a graphical programming language, which is an advanced development platform which has the functions of the data acquisition, the monitoring and the data analysis from NI Company in USA.

\section{System Description}

Acquisition System. First of all, we should achieve the original BCG signal. According to its measurement principle, our laboratory designed and realized a new sitting BCG signal detecting device. Fig. 1 is the experiment environment.

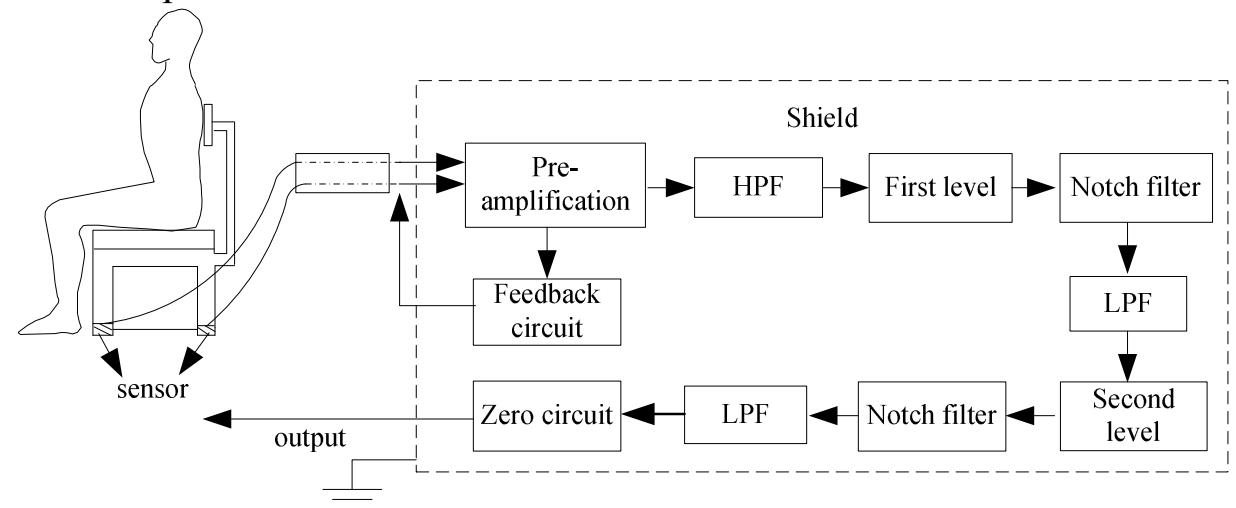

Fig. 1. The measurement system 
Sensor circuit is a home chair refitted by resistance strain weighing sensors used in electronic weight scale. And the body vibration is transformed into electrical signal by the pressure sensor. The electrical signal is put into high stable, high common-mode rejection ratio pre-amplification difference circuit. While the pre-amplifier's input is feed back to the sensor network through the feedback circuit to reduce common-mode noise. The preamplifier's output is put into high-passed filter to remove direct-current signal and avoid the following amplifier's output saturation. Notch Filter is used to remove power frequency disturbance. After the amplification of the first and the second level, BCG signal is obtained at the output port. The second level amplifier's amplification is able to be adjusted to obtain more ideal BCG signal. BCG signal is put into Low-pass filter to eliminate high frequency noise further more. The amplifier and the filter circuit are both in the shielding layer to shield the outside electromagnetic disturbance. The original BCG is transferred to PC by Bluetooth device.

Analysis System. Signals recorded during BCG chair are highly nonstationary and noisy, mainly due to muscular activity and motion artifacts. So we need to design a automatic analysis system to satisfy the real-time monitor at home. The system diagram is showed in Fig.2.

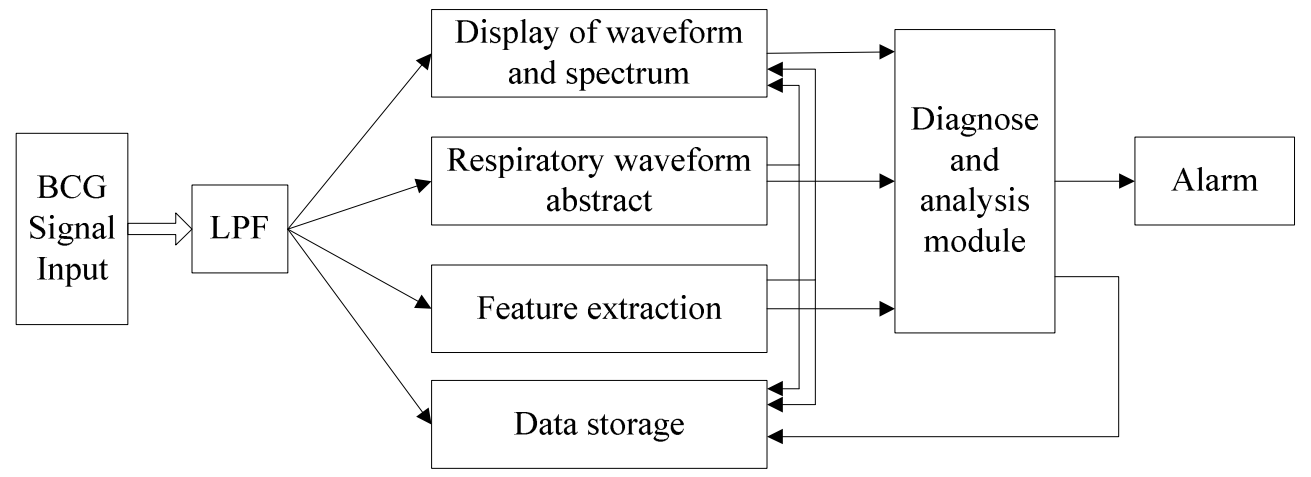

Fig. 2. Analysis system block diagram

Remove of human and environmental noise by LPF. Then display the filtered wave. At the same time, abstract the respiratory component, extract the waveform feature points, and store the data. A diagnose module is used to judge the physiological status and to arouse the alarm when crisis happens.

\section{Algorithms and Methods}

Filter Algorithm. Apply wavelet transform adaptive de-noising algorithm to realize PLF. There are two channels in time domain LMS adaptive de-noising. One is main channel, which receives the superposition of useful signal $s$ and the interference $r_{0}$. The other is reference channel, which receives the interference $r$. Because of the different transmission paths, although $r$ and $r_{0}$ are from the same noise source, they aren't same. But they are correlation and independent of the useful signal. It meets adaptive filter condition. The output of the system is fed from error signal, so:

$$
e=d-y=s+r_{0}-y
$$

Adaptive filter should adjust its weight vectors to make $y$ close to $r_{0}$, on the condition of least mean square error. Then the signal-to-noise ratio of the output signal is improved. The convergence speed of time domain LMS algorithm is easy to be affected by the condition number of input signal's autocorrelation matrix. Orthogonal wavelet transform segments the original signal in frequency domain, removes correlation and improves the convergence speed of LMS algorithm. It also changes the serial arithmetic to parallel arithmetic, and improves the computing speed. According to MALLAT algorithm, one N points discrete signal's J layers wavelet decomposition is used in this chapter [2]. 
Respiratory Detection. A biological series is often the result of combined contributions from different sources. The adaptive filtering is a powerful tool to separate the contribution of interest signal from other contributions. The applications of this methodology can be classified in two schemes: noise canceller and signal enhancer. The former is applied when, besides the previous series, a new series is available containing components correlated with the noise but not with the signal. This filter is able to cancel the correlated noise and the output will not suffer from any distortion. The latter is applied when a new series is available containing components correlated with the signal but not with the noise; this filter enhances the signal related components. In this case, the signal passing through the filter can suffer of some distortion [3].

The adaptive interference canceling is an attractive feature of an adaptive filter. This approach is viable only when an additional reference input is available containing noise $n_{1}$, which is correlated with the original corrupting noise $n_{0}$. The adaptive filter receives the reference noise, filters it, and subtracts the result from the noisy primary input, $s+n_{0}$. For this adaptive filter, the noisy input $s+n_{0}$ acts as the desired response. The system output acts as the error for the adaptive filter. Adaptive interference cancellation generally performs much better than the classical approach, since the noises is subtracted out rather than filtered out [4].

A BCG signal without breath was used to provide a clear recording of the maternal heartbeat which served as the reference input. The BCG signal with breath of subject served as the primary input. This reference input is adaptively filtered and subtracted from the heartbeat signal. All signals are filtered and digitized. The irrelevant interference component will be filtered at the same time. However, the frequency derived of respiratory is close and lower than the heartbeat, so the envelope detection algorithm is needed to demodulate the output signal. In this paper, we choose the multiple derivation and peak extraction method to draw the envelope of the signal. And the recovery waveform is the estimate of respiratory component.

Feature Extraction. The Hamilton-Tompkins algorithm [5], applies a squaring function to rectify the differentiated BCG. The squaring function provides further attenuation of other BCG features, leaving the $\mathrm{W}$ complex as outstanding positive peaks in the signal regardless of their polarity in the original BCG recording. The transform can also be viewed as a measure of energy with a threshold that verifies if the output is enough to carry the energy of the $\mathrm{W}$ complex.

The differentiation formula as implemented in the original method is:

$$
v_{r}[n]=\frac{1}{8}(2 x[n]+x[n-1]-x[n-3]-2 x[n-4])
$$

The five-point derivative prevents high-frequency noise amplification, in the present implementation high-frequency noise is further attenuated by the Kaiser Window filter [6]. Peaks are found by comparing the time-averaged signal to a primary threshold, derived from the threshold coefficient and the amplitude of previous peaks. The VI block diagram is shown as Fig.3.

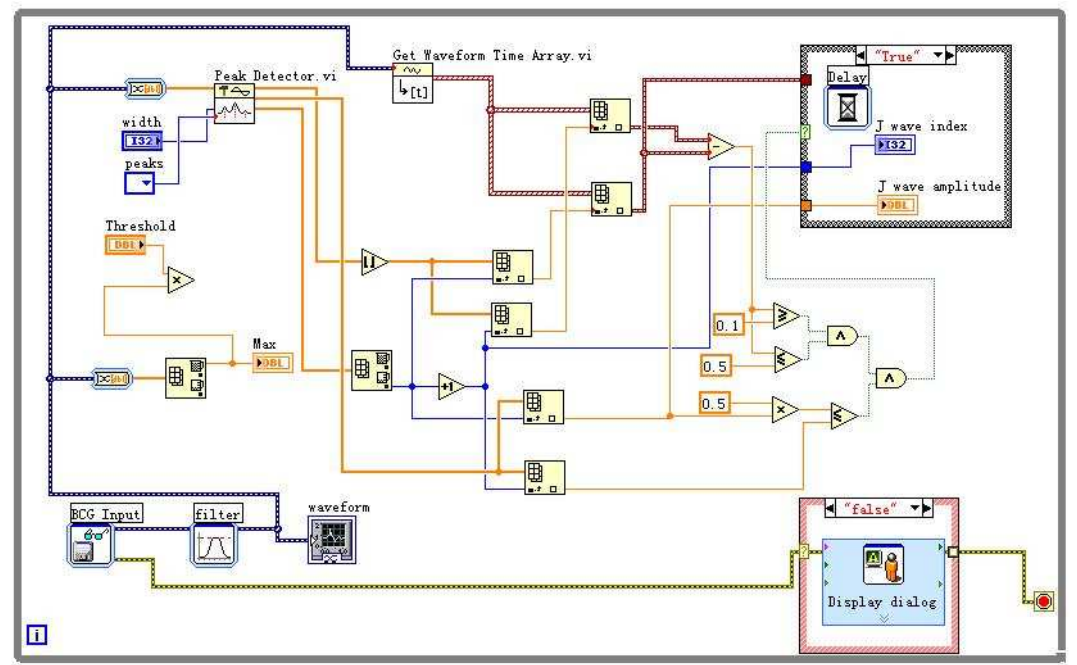

Fig. 3. The VI block diagram of feature extraction algorithm 
Data Storage. LabVIEW provides the function to store a variety of file format, such as text format, Excel, binary file format, and $\mathrm{G}$ langue data $\log$ file. At the same time, the other functions, including information storage, management and file compression are provided by the system.

\section{Simulation Results}

By summing up the algorithms above, we design an integral monitoring system through LabVIEW plat. Fig. 4 is the automatic analysis system of BCG signal.

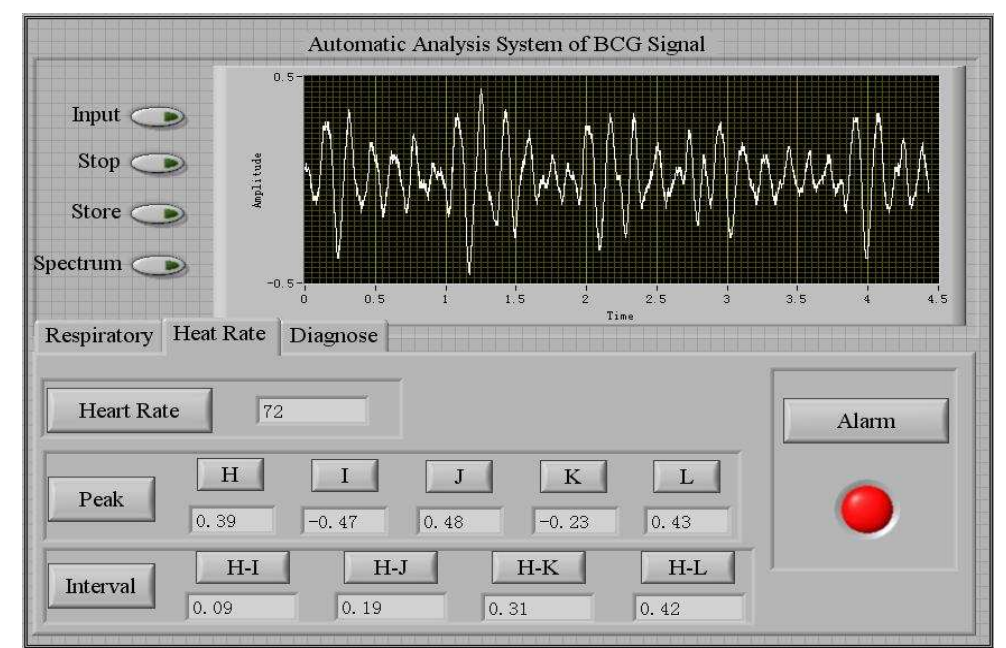

Fig. 4. Simulation interface of automatic analysis system of BCG signal

In this interface, the upper part is in charge of reading the BCG signal, storing the data, and displaying the waveform and spectrum of the cardiac cycle component and respiratory component. The lower half applies different tabs to analyze various features, diagnose the state of heart function and respiratory, and to alarm timely.

\section{Conclusion}

This paper designed and realized a system to record and automatic analyze the BCG signal based on virtual instruments, which can provide visual interface to comfort the user to monitoring heart and lung real-time. More algorithms and methods could be used in this system to improve the system performance.

\section{Acknowledgements}

Thanks for the support of the Fundamental Research Funds for the Central Universities (N100604006).

\section{References}

[1] J.W. Huang, Automatic analysis system of ECG by LabVIEW, first ed., Yunnan University Press, Kunming, 2009.

[2] J.J. Jin, X Wang, S.L. Li, Wavelet Transform adaptive de-noising algorithm and application based on a novel variable step function, Second International Workshop and Knowledge Discovery and Data Mining. 1 (2009) 80-83.

[3] M. Varanini, M. Emdin, F. Allegri, Adaptive filtering of ECG signal for deriving respiratory activity, Proc. 4th Annu. Allerton Conf. Circuits and Systems Theory. (1991) 621-624.

[4] B. Widrow, M. A. Lehr, Noise canceling and channel equalization, in Conf. Rec. 1995 IEEE Int. Conf. Communications. (1995) 648-650.

[5] N.M. Arzeno, Z.D. Deng, C.S. Poon, Analysis of first-derivative based QRS detection algorithms, IEEE transactions on Biomedical Engineering, 55 (2008) 478-484.

[6] A. Ligtenberg, M. Kunt, A robust-digital QRS-detection algorithm for arrhythmia monitoring, Comput. Biomed. Res., 16 (1983) 273-286. 